Nuclear Physics B116 (1976) 133-140

(c) North-Holland Publishing Company

\title{
ON THE BOUND STATES OF THE MASSLESS FREE FIELD IN TWO SPACE-TIME DIMENSIONS
}

\author{
Rudolf SEILER * \\ Institut für Theoretische Physik, Freie Universität Berlin, Germany
}

Dietrich A. UHLENBROCK †

Department of Mathematics, University of Wisconsin, Madison, Wisconsin 53706

David N. WILLIAMS

Harrison M. Randall Laboratory of Physics, The University of Michigan, Ann Arbor, Michigan 48109

Received 31 May 1976

\begin{abstract}
We point out the peculiar kinematics of zero-mass fields in two space-time dimensions by showing that the $n$-fold tensor product of one-particle representations of the Poincaré group for $n \geqslant 2$ contains a countable infinity of discrete, zero-mass representations. We relate this result to the well-known "bound" states in every charge sector of the fermion Fock space.
\end{abstract}

\section{Introduction}

In the early days of quantum field theory the existence of a massless boson (photon) as a bound state of free massless fermions (neutrino) was expected to be a general fact, independent of dimension [1]. By now it is clear that two-dimensional quantum field theory of massless particles is special for kinematical reasons, since there are five mass-zero orbits of the connected component of the Poincaré group in $\mathrm{R}^{2}$.

It is known that there is no scalar, free massless boson field in two dimensions [2]. On the other hand, there do exist zero-mass scalar particle states in the Fock space of a free massless fermion field (see ref. [1] and footnote 10 of ref. [3]). Coleman has pointed out that this situation is a result of the two-dimensional kinematics.

In sect. 2 we analyze the problem of the existence of massless scalar "bound"

\footnotetext{
* Supported in part by grant DFG SE $287 / 2$.

Supported in part by grants NSF MPS-75-06366, MPS-74-22844.
} 
particles in a group theoretical setting. It is shown that any $n$-fold, $n \geqslant 2$, tensor power of a one-particle representation of the Poincare group with mass zero has not only a continuous mass spectrum starting at the two or more particle threshold (zero in this case), familiar for four-dimensional space-time dimensions, but also an extra, discrete contribution to the mass spectrum at zero mass, corresponding to a countably infinite, orthogonal direct sum of one-particle representations. These results are independent of statistics. The intuitive explanation is that the set of $n$ particle momenta for which the particles travel "together" (with the same speed) has non-zero measure in $n$-particle momentum space if and only if the masses are zero and space has dimension one ${ }^{\star}$.

In sect. 3 we consider the Fock space $\mathcal{F}$ of the free massless fermion field. We explicitly identify the eigenspace $\chi$ of the mass operator $M$ with eigenvalue zero. $\chi$ decomposes as a direct sum of unitarily equivalent subspaces, each one belonging to a different charge sector. It is shown that the application of the current on the vacuum produces a single zero-mass boson state and contains no continuum contribution. Since the algebra of the currents is irreducible in every charge sector [4], we conclude that higher powers of the current on the vacuum produce the remaining countable infinity of bosons, plus the continuum.

\section{Tensor products of one-particle representations}

The Hilbert space for a single, zero-mass particle of helicity $\sigma$ is

$$
\mathscr{H}_{1}=L_{2}\left(\mathbb{R} \backslash 0, \mathrm{~d} k^{s} / 2 \omega\right),
$$

where $k^{s}$ is the space component of the two-vector $k=\left(\omega, k^{s}\right), \omega=\left|k^{s}\right|$.

The identity component of the Poincaré group, $\mathscr{P}_{\mathrm{I}}$, consists of two-dimensional translations, and proper, homogeneous Lorentz transformations:

$$
(a, \Lambda) x=\Lambda x+a
$$

where $x, a \in \mathbb{R}^{2}$, and

$$
\begin{aligned}
& \Lambda x=\left(\begin{array}{l}
x^{0} \cosh \lambda+x^{s} \sinh \lambda \\
x^{0} \sinh \lambda+x^{s} \cosh \lambda
\end{array}\right), \\
& -\infty<\lambda<\infty .
\end{aligned}
$$

\footnotetext{
* Notice that existence versus non-existence of free massless scalar fields and massless particles in an $n$-fold tensor product of representations of the Poincaré group as a function of space dimension $s$ goes in the opposite direction.
} 
In terms of light-cone variables,

$$
x_{ \pm}=\frac{1}{2}\left(x^{0} \pm x^{s}\right), \quad(\Lambda x)_{ \pm}=\mathrm{e}^{ \pm \lambda} x_{ \pm} .
$$

The one-particle, unitary representation of $\mathcal{P}_{I}$ for helicity $\sigma$ has the action on $\mathscr{H}_{1}$ :

$$
U_{1, \sigma}(a, \Lambda) f(k)=\mathrm{e}^{i a \cdot k} \mathrm{e}^{i \sigma \lambda} f\left(\Lambda^{-1} k\right) .
$$

This representation has two irreducible components, corresponding to the values of the proper Lorentz-invariant sgn $k^{s}$.

Now consider the tensor product representation $U_{2}=U_{1, \sigma_{1}} \otimes U_{1, \sigma_{2}}$, on $\mathscr{K}_{2}=$ $\mathscr{K}_{1} \otimes \mathscr{K}_{1}$. We ask the question: is there a subset of non-zero $\left(\mathrm{d} k_{1}^{s} \mathrm{~d} k_{2}^{s}\right)$ measure corresponding to total mass zero, namely

$$
\begin{aligned}
0 & =\left(k_{1}+k_{2}\right) \cdot\left(k_{1}+k_{2}\right)=2 k_{1} \cdot k_{2} \\
& =2\left(\omega_{1} \omega_{2}-k_{1}^{s} k_{2}^{s}\right)=2\left(\left|k_{1}^{s} k_{2}^{s}\right|-k_{1}^{s} k_{2}^{s}\right) .
\end{aligned}
$$

The answer is yes, the subset sgn $k_{1}^{s} k_{2}^{s}>0$, which occupies two quadrants of the $\left(k_{1}^{s}, k_{2}^{s}\right)$ plane. Let us call these two quadrants $K_{+}$, and the remaining two quadrants, where $\operatorname{sgn} k_{1}^{s} k_{2}^{s}<0, K_{-}$.

Note that $K_{+}$is the set of two-particle momenta where the particles have the same velocity.

We may split $\mathscr{x}_{2}$ :

$$
\mathscr{X}_{2}=\mathscr{X}_{+} \oplus \mathscr{X}_{-} \text {, }
$$

where $\mathscr{X}_{ \pm}=\left\{f \in \mathscr{X}_{2}\right.$ : support $\left.f \subset K_{ \pm}\right\}$. Then $\mathscr{K}_{+}$consists of eigenvectors of the mass operator with zero mass, and $\mathscr{K}_{-}$is the continuum subspace of the mass operator.

We may further split $\mathscr{X}_{+}$into one-particle representations after choosing appropriate variables. These we take to be the spatial component of the total momentum,

$$
k^{s}=k_{1}^{s}+k_{2}^{s},
$$

then $\omega=\left|k^{s}\right|=\omega_{1}+\omega_{2}$, and the Lorentz-invariant ratio

$$
\alpha=\theta\left(k_{1}^{s}\right) \frac{k_{1,+}}{k_{2,+}}+\theta\left(-k_{1}^{s}\right) \frac{k_{1,-}}{k_{2,-}}=\frac{k_{1}^{s}}{k_{2}^{s}} .
$$

The old variables in terms of the new are

$$
k_{1}^{s}=\frac{k^{s}}{1+\alpha^{-1}}, \quad k_{2}^{s}=\frac{k^{s}}{1+\alpha},
$$




$$
\begin{aligned}
& -\infty<k^{s}<\infty, \quad 0<\alpha<\infty, \\
& \frac{\mathrm{d} k_{1}^{s} \mathrm{~d} k_{2}^{s}}{2 \omega_{1} 2 \omega_{2}}=\frac{\mathrm{d} k^{s} \mathrm{~d} \alpha}{2 \omega 2 \alpha} .
\end{aligned}
$$

Thus, we may write

$$
\begin{aligned}
\mathscr{H}_{+} & =L_{2}\left(\frac{\mathrm{d} k^{s}}{2 \omega}\right) \otimes L_{2}\left(\frac{\mathrm{d} \alpha}{\alpha}\right), \\
& =\mathscr{H}_{1} \otimes L_{2}\left(\frac{\mathrm{d} \alpha}{\alpha}\right),
\end{aligned}
$$

and the action of the group may be written

$$
U_{2,+}(a, \Lambda) f(k, a)=\mathrm{e}^{i k \cdot a} \mathrm{e}^{i\left(\sigma+\sigma^{\prime}\right) \lambda} f\left(\Lambda^{-1} k, \alpha\right),
$$

i.e.,

$$
U_{2,+}=U_{1, \sigma+\sigma^{\prime}} \otimes 1
$$

Clearly, any orthogonormal basis in the $\alpha$ Hilbert space induces a splitting of $\mathscr{X}_{+}$ into a countable orthogonal direct sum of copies of $\mathscr{x}_{1}$, and of $U_{2,+}$ into a direct sum of one-particle representations.

It is very easy to check that zero mass and one-space dimension is the only case where a subset of two-particle momenta with fixed total mass has non-zero measure. In all other cases, the two-particle Hilbert space has no discrete mass eigenstates.

Note that identical particle symmetrization or antisymmetrization simply induces the symmetries $f\left(k^{s}, \alpha\right)= \pm f\left(k^{s}, \alpha^{-1}\right)$, and the phenomenon persists.

If we go now to $n$ particles, we can either proceed by pairwise reduction on the subspace of functions having all spatial momenta of the same sign, or we can argue directly that the set of $\left(k_{1}^{s}, \ldots, k_{n}^{s}\right)$ corresponding to zero total mass is again of nonzero measure:

$$
0=\left(k_{1}+\ldots+k_{n}\right)^{2}=\sum_{i<j} 2 k_{i} \cdot k_{j} \Leftrightarrow \operatorname{sgn} k_{i}^{s}=\operatorname{sgn} k_{j}^{s} \quad \text { for all } i \text { and } j .
$$

The discrete, zero-mass states are, as for $n=2$, the vectors supported at momenta where either all the particles travel to the right with the speed of light, or they all travel to the left with the same speed.

\section{Zero-mass subspace of the massless fermion Fock space}

For convenience we use the box cutoff of the fermion field theory. The box cutoff avoids infrared problems. In the infinite volume limit an analogous analysis is 
possible if one allows for an infrared cut-off in the scalar potential (see below) à la Klaiber [6] .

Let $\psi(x)$ be the massless fermion field in two space-time dimensions with box cut-off $L$

$$
\begin{aligned}
& \psi(x)=\frac{1}{\sqrt{L}} \sum_{\kappa}\left(\mathrm{e}^{i \kappa \cdot x} u(\kappa) a(\kappa)+\mathrm{e}^{-i \kappa \cdot x} u_{\mathrm{c}}(\kappa) b^{*}(\kappa)\right) \\
& \kappa=\left(\kappa_{0}=|\kappa|, \kappa\right), \quad \kappa=\frac{2 \pi}{L}\left(n-\frac{1}{2}\right), \quad u_{\mathrm{c}}=\gamma^{5} u, \quad n \in Z, \\
& u(\kappa)=\left(\begin{array}{c}
-\theta(-\kappa) \\
\theta(\kappa)
\end{array}\right) .
\end{aligned}
$$

The two-point functions of $\psi$ are given by

$$
\begin{aligned}
& \left\langle\psi_{\tau}(\bar{f}) \psi_{\tau^{\prime}}^{*}(g)\right\rangle=\delta_{\tau \tau^{\prime}} \sum_{\tau \kappa>0} \overline{\widetilde{f}}(k) g(\kappa) \\
& =\left\langle\psi_{\tau}^{*}(\bar{f}) \psi_{\tau}(g)\right\rangle \delta_{\tau \tau^{\prime}}, \quad \tau, \tau^{\prime}= \pm 1,
\end{aligned}
$$

where the bar denotes Fourier transformation

$$
\bar{f}(k)=\frac{1}{2 \pi} \int \mathrm{d}^{2} x \mathrm{e}^{-i k \cdot x} f(x)
$$

and $\bar{f}(\kappa)=\bar{f}(|\kappa|, \kappa)$. It follows from (1) that the one-particle and antiparticle Hilbert space decompose into direct sums of two spaces

$$
\mathscr{X}^{\mathrm{p}}=\mathscr{X}_{+}^{\mathrm{p}} \oplus \mathscr{X}_{-}^{\mathrm{p}}, \quad \mathscr{X}^{\mathrm{a}}=\mathscr{X}_{+}^{\mathrm{a}} \oplus \mathscr{K}_{-}^{\mathrm{a}} .
$$

Hence the Fock space $\mathcal{F}$ of the massless fermion field is isomorphic to the direct product of four factors

$$
\mathcal{F}=\mathcal{F}_{+}^{\mathrm{p}} \otimes \mathcal{F}_{-}^{\mathrm{p}} \otimes \mathcal{F}_{+}^{\mathrm{a}} \otimes \mathcal{F}_{-}^{\mathrm{a}},
$$

where $\mathcal{F}_{+}$is the Fock space over $\mathscr{K}_{+}^{\mathrm{p}}$, etc. In particular, $\mathcal{F}$ is isomorphic to the product of $\mathcal{F}_{+}$and $\mathcal{F}_{-}, \mathcal{F}=\mathcal{F}_{+} \otimes \mathcal{F}_{-}$where $\mathcal{F}_{ \pm}=\mathcal{F}_{ \pm}^{\mathrm{p}} \otimes \mathcal{F}_{ \pm}^{\mathrm{a}}$.

The light-cone combinations of the fermion current $j^{\mu}=: \psi^{+} \gamma^{\mu} \psi: \psi^{+}=\psi^{*} \gamma^{0}$

$$
\gamma^{0}=\left(\begin{array}{ll}
0 & 1 \\
1 & 0
\end{array}\right), \quad \gamma^{1}=\left(\begin{array}{cc}
0 & 1 \\
-1 & 0
\end{array}\right)
$$

can be written in terms of a scalar field $\chi=\phi_{-}+\phi_{+}$,

$$
\phi_{\tau}(x)=\frac{1}{\sqrt{L}} \sum_{\tau \kappa}|k|^{-1 / 2}\left(\mathrm{e}^{-i k \cdot x} c(k)+\mathrm{e}^{i k \cdot x} c^{*}(k)\right), \quad k=\frac{2 \pi}{L} n,
$$




$$
j_{\tau}=\frac{1}{2}\left(j^{0}+\tau j^{1}\right), \quad j_{\tau}(x)=\frac{1}{\sqrt{2 \pi}} \frac{\mathrm{d}}{\mathrm{d} x^{0}} \phi_{\tau}(x)+\frac{1}{L} Q_{\tau}
$$

The $c$ 's are boson operators which are bilinear in the fermion operators

$$
\begin{aligned}
& c(k)=i\left(\frac{2 \pi}{L|k|}\right)^{1 / 2} \sum_{\kappa}\left\{\theta(\kappa k)\left[b^{*}(\kappa) b(\kappa+k)-a^{*}(\kappa) a(\kappa+k)\right]\right. \\
& \quad+\theta(\kappa(k-\kappa)) a(k-\kappa) b(\kappa)\}^{-}
\end{aligned}
$$

and $Q_{\tau}$ are the light cone combinations of the charge operators:

$$
Q_{\tau}=\sum_{\tau \kappa>0}\left(a^{*}(\kappa) a(\kappa)-b^{*}(\kappa) b(\kappa)\right) .
$$

The representation of the boson field $\phi$ decomposes according to the charge structure of $\mathcal{F},\left[Q_{\tau}, \phi\right]=0$. Furthermore in every charge sector the representation is Fock [4] . Notice that $\phi_{\tau}$ and $Q_{\tau}$ act only in a non-trivial manner on $\mathcal{F}_{\tau}$.

Now we are prepared to state the following

Theorem: Let $\mathcal{F}_{\tau}, \tau= \pm 1$, be the fermion Fock space over the Hilbert space $\mathscr{K}_{\tau}^{\mathrm{p}} \oplus \mathscr{H}_{\tau}^{\mathrm{a}}$. By the natural injection they can be considered as linear subspaces of the fermion Fock space $\mathcal{F}$. Let $M$ be the mass operator on $\mathcal{F}$. Then the space $\boldsymbol{X}=$ $\mathcal{F}_{+} \oplus \mathcal{F}_{-}$is the eigenspace of $M$ with eigenvalue zero. The spectrum of $M$ on the orthogonal complement $\chi^{1}$ is absolutely continuous. Furthermore $x^{\text {is the direct }}$ sum of unitarily equivalent subspaces in the different charge sectors

$$
\begin{array}{ll}
\mathcal{F}=\underset{n, m}{\oplus} \mathcal{F}_{n m}, \quad & \Re_{n, m}^{\oplus} \Re_{n m}, \quad \chi_{n m}=\mathcal{F}_{+, n} \oplus \mathcal{F}_{-, n}, \\
Q_{\tau} \mathcal{F}_{\tau, n}=n \mathcal{F}_{\tau, n}, & n \text { integer } .
\end{array}
$$

Proof: $X$ is an eigenspace of $M^{2}$ for the point eigenvalue zero: The square of the mass operator is defined by

$$
M^{2}=P_{0}^{2}-P_{1}^{2}, \quad P_{\mu}=\sum_{\kappa} \kappa_{\mu}\left(a^{*}(\kappa) a(\kappa)+b^{*}(\kappa) b(\kappa)\right), \quad \mu=0,1,
$$

and essentially self-adjoint on the set of states with finite modes. Every element of $\mathcal{F}_{+}$can be approximated by a finite linear combination of the states

$$
\prod_{\substack{i \in I \\ e \in L}} a^{*}\left(k^{i}\right) b^{*}\left(\kappa^{\prime} e\right) \Omega, \quad I, L \text { finite sets } \kappa^{i}>0, \quad \kappa^{\prime} e>0,
$$

and since on those states $P_{0}$ equals $P_{1}$ the mass operator vanishes. A similar argument holds for $\mathcal{F}_{-}$. We leave to the reader the exercise of showing the converse statement.

$\varkappa$ is a direct sum of unitarily equivalent subspaces: in ref. [4] unitary operators 
$U_{\tau}$ have been constructed mapping $\mathcal{F}_{\tau, n}$ onto $\mathcal{F}_{\tau, n-1}$. On the other hand, if $Q_{\tau}$ (restricted to $\mathscr{F}_{\tau}$ ) has the spectral decomposition $Q_{\tau}=\Sigma_{n \in Z} P_{\tau, n}$ then $\mathscr{F}_{\tau, n}=$ $P_{\tau, n} \mathcal{F}_{\tau}$. Hence the statement follows.

In order to relate the results of this section with the group theoretical analysis we compute the wave function in the two-fermion subspace of the Fock space generated by the current $j_{\tau}$ from the vacuum,

$$
j_{\tau}(f) \Omega=\frac{1}{L} \sum_{\substack{\tau\left(\kappa^{\prime}+\kappa\right)>0 \\ \kappa^{\prime} \kappa>0}} \tilde{f}\left(\left|\kappa^{\prime}+\kappa\right|, \kappa+\kappa^{\prime}\right) a^{*}(\kappa) b^{*}\left(\kappa^{\prime}\right) \Omega .
$$

Hence the wave functions do have their support in $K_{+}$. They are however quite special, in fact they are constant along the straight lines $\kappa+\kappa^{\prime}=$ constant in the $\kappa, \kappa^{\prime}$ plane. Notice that the wave function of $j_{+}(f) \Omega$ and $j_{-}(f) \Omega$ have support in the first and the third quadrants respectively. The reader might convince himself that the wave function in the two-particle fermion space which belongs to a state $\Pi_{i=1}^{N} j_{\tau}\left(f_{i}\right)$, $N>2$, do in general depend on the sum and the difference of the momenta. We should like to emphasize however that not every bilinear expression in the fermion field maps the vacuum into a discrete zero mass state of the two-particle subspace. Consider for instance the scalar Wick monomial

$$
: \bar{\psi} \psi:(f)=: \psi_{+}^{*} \psi_{-}:(f)+: \psi_{-}^{*} \psi_{+}:(f),
$$

which does not commute with $Q_{\tau}, \tau= \pm 1$. The two-particle wave function of this operator is

$$
\frac{2 \pi}{L} f\left(\kappa_{1}+\kappa_{2}\right)\left(\theta\left(\kappa_{1}\right) \theta\left(-\kappa_{2}\right)+\theta\left(-\kappa_{1}\right) \theta\left(\kappa_{2}\right)\right),
$$

and obviously in the subspace of the two-fermion subspace belonging to the continuous spectrum of the mass operator (second and fourth quadrant in the $k_{1}, k_{2}$ plane).

Finally, we should like to comment on footnote 10 of ref. [3]. Coleman has noticed that the amplitude $\left\langle: \bar{\psi} \psi:(x) j_{\tau}(f)\right\rangle$ of the discrete eigenstate $j_{\tau}(f) \Omega$ with respect to the Wick polynomial $: \bar{\psi} \psi:$ vanishes. Our analysis shows the basic reason: one vector belongs to the continuum part of the mass operator, the other of the point spectrum. On the other hand, the amplitude $\left\langle: \psi_{\tau}^{*} \psi_{\tau}:(x) j_{\tau}(f)\right\rangle$ does not vanish. In fact the two-point function of : $\psi_{+}^{*} \psi_{+}:(x)$ is singular for $x \sim 0$ (for easy comparison with ref. [3] we use the infinite volume formalism [5])

$$
\left\langle: \psi_{+}^{*} \psi_{+}:(x): \psi_{+}^{*} \psi_{+}:(0)\right\rangle=\left(\frac{1}{2 \pi i}\right)^{2}\left(\frac{1}{x^{0}-x^{1}-i 0}\right)^{2} .
$$

However the field : $\psi_{+}^{*} \psi_{+}:(x)$ which creates scalar, massless boson particles out of the vacuum is not a scalar field! 
The first named author should like to express his appreciation for the hospitality to A. Wightman, and to Princeton University for financial support.

\section{References}

[1] M. Born and N.S. Nagandra Nath, Proc. Ind. Acad. Sci 3 (1936) 318.

[2] A.S. Wightman, Cargèse lectures 1964.

[3] S. Coleman, Comm. Math. Phys. 31 (1973) 259.

[4] D.A. Uhlenbrock, Comm. Math. Phys. 4 (1967) 64.

[5] B. Klaiber, Boulder lectures 1967, Lectures in Theoretical Physics (Gordon and Breach, New York, 1968). 